\title{
Identification Problem of Source Term of A Reaction Diffusion Equation
}

\author{
Bo Zhang \\ Linyi University at Feixian \\ Feixian, Shandong, P.R.China
}

\begin{abstract}
This paper will give the numerical difference scheme with Dirichlet boundary condition, and prove stability and convergence of the difference scheme, final numerical experiment results also confirm effectiveness of the algorithm.
\end{abstract}

Keywords- Fractional derivative; Numerical difference scheme; The gradient regularization method.

\section{INTRODUCTION}

Source term inversion in groundwater pollution is a class of inverse problems[7,8], and is also important field of inverse problem research. Scholars have done a large amount of work and obtained many results. Reference [2] presented a new gradient regularization algorithm to solve an inverse problem of determining source terms in one-dimension solute transport with final observations, and reference [3] proposed a implicit method to solve a class of space-time fractional order diffusion equations with variable coefficient.

However, when fractional derivative replaces second derivative in diffusion equations, there is anomalous diffusion phenomenon. In this paper, we give the numerical difference scheme in source term identification with Dirichlet boundary condition, and we prove the stability and convergence of the difference scheme, also verify the practicality and effectiveness of the algorithm through numerical experiment.

In this paper, we use difference scheme to solve the forward problem, and when solving the inverse problem, we use the gradient regularization method based on Tikhonov regularization strategy. Here, the additional information for source term identification is set as the final observations, and suppose that the source term function are only concerned with the space variable and has nothing to do with the time variable.

In fact, the solute transport model can be described by the following equation[1]

$$
\begin{aligned}
\frac{\partial y}{\partial t}= & u(x) \frac{\partial^{2} y(x, t)}{\partial x^{2}}-v(x) \frac{\partial y(x, t)}{\partial x}-c(x) y(x, t) \\
& +b(x, t), \quad x \in[0, L], t \in[0, T]
\end{aligned}
$$

(1)

by introducing fractional derivative and adding initial boundary conditions[5], Eqs. (1) will be modified as the following problem

$$
\begin{array}{cc}
\frac{\partial^{\alpha} y}{\partial t^{\alpha}}=u(x) \frac{\partial^{\beta} y(x, t)}{\partial x^{\beta}}-v(x) \frac{\partial y(x, t)}{\partial x}-c(x) y(x, t) \\
+b(x, t), & x \in \Omega, t>0, \\
D y(x, t)=g(x, t), & x \in \Omega, t>0, \\
\operatorname{Ey}(x, 0)=f(x), & x \in \Omega,
\end{array}
$$

where $0<\alpha<1,1<\beta<2, D$ represents matrix operator with boundary condition, $E$ represents matrix operator with initial condition, $y(x, t)$ and $b(x)$ represent undetermined source term function and undetermined vector function respectively.

Inverse problem of this problem is to determine unknown vector function $b(x)$ by Eqs. (2)-(4) and the additional condition below

$$
\left.y(x, t)\right|_{x=T}=\psi(x) .
$$

\section{NUMERICAL DIFFERENCE SCHEME}

Considering the following space-time reaction diffusion equations

$$
\begin{aligned}
\frac{\partial^{\alpha} y(x, t)}{\partial t^{\alpha}}= & u(x) \frac{\partial^{\beta} y(x, t)}{\partial x^{\beta}}-v(x) \frac{\partial y(x, t)}{\partial x} \\
& -c(x) y(x, t)+b(x, t),
\end{aligned}
$$

(6)

(7)

$$
y(x, 0)=f(x),
$$

$$
y(0, t)=g_{1}(t), y(L, t)=g_{2}(t) .
$$

Where $u(x)>0, v(x)>0, c(x) \geq 0$ are continuous functions on $[0, L], g_{1}(t)>0, g_{2}(t)>0$ are continuous functions on $[0, T], b(x, t)$ is continuous on $[0, L] \times$ $[0, T], \alpha \in(0,1), \beta \in(1,2), \frac{\partial^{\alpha} y(x, t)}{\partial t^{\alpha}}, \frac{\partial^{\beta} y(x, t)}{\partial x^{\beta}} \quad$ are 
Caputo fractional derivative and Riemann-Liouville fractional derivative respectively[5]

$$
\frac{\partial^{\alpha} y(x, t)}{\partial t^{\alpha}}=\frac{1}{\Gamma(1-\alpha)} \int_{0}^{t}(t-\eta)^{-\alpha} \frac{\partial y(x, \eta)}{\partial \eta} d \eta
$$

(9)

$$
\frac{\partial^{\beta} y(x, t)}{\partial x^{\beta}}=\frac{1}{\Gamma(2-\beta)} \frac{\partial^{2}}{\partial x^{2}} \int_{0}^{t} \frac{y(\xi, t)}{(x-\xi)^{\beta-1}} d \xi .
$$

Suppose that Eqs. (6)-(8) has a unique and smooth enough solution. $\tau=T / n$ is time step, $\Delta x=h=L / m$ is space $\quad$ step, $\quad t_{k}=k \tau(k=0,1,2, \mathrm{~L}, n)$, $x_{i}=i h(i=0,1,2, \mathrm{~L}, \quad m)$. For the time fractional derivative, we usually adopt following finite difference approximation

$$
\begin{aligned}
& \frac{\partial^{\alpha} y\left(x_{i}, t_{k+1}\right)}{\partial t^{\alpha}} \\
= & \frac{1}{\Gamma(1-\alpha)} \sum_{j=0}^{k} \frac{y\left(x_{i}, t_{j+1}\right)-y\left(x_{i}, t_{j}\right)}{\tau} \int_{j \tau}^{(j+1) \tau} \frac{d \xi}{\left(t_{k+1}-\xi\right)^{\alpha}}+\tau \\
= & \frac{\tau^{1-\alpha}}{\Gamma(2-\alpha)} \sum_{j=0}^{k} \frac{y\left(x_{i}, t_{k+1-j}\right)-y\left(x_{i}, t_{k-j}\right)}{\tau}\left[(j+1)^{1-\alpha}-\right. \\
& \left.j^{1-\alpha}\right]+O(\tau),
\end{aligned}
$$

(11)

$$
\frac{\partial y\left(x_{i}, t_{k+1}\right)}{\partial x}=\frac{y\left(x_{i}, t_{k+1}\right)-y\left(x_{i-1}, t_{k+1}\right)}{h}+O(h) .
$$

For $\frac{\partial^{\beta} y(x, t)}{\partial x^{\beta}}$, by using Grünwald's improved formula [4], we have that

$$
\frac{\partial^{\beta} y\left(x_{i}, t_{k+1}\right)}{\partial x^{\beta}}=\frac{1}{h^{\beta}} \sum_{j=0}^{i+1} a_{j} y\left(x_{i}-(j-1) h, t_{k+1}\right)+O(\tau+h),
$$

where

$$
\begin{aligned}
& a_{0}=1, a_{1}=-\beta, a_{j}=(-1)^{j}\left(\begin{array}{c}
\beta \\
j
\end{array}\right), \\
& \left(\begin{array}{c}
\beta \\
j
\end{array}\right)=\beta(\beta-1) \mathrm{L}(\beta-j+1) /(j !), j=1,2,3, \mathrm{~L} .
\end{aligned}
$$

Substituting Eqs. (11)-(13) into Eqs. (6)-(8), we obtain

$$
\begin{aligned}
& \frac{\tau^{1-\alpha}}{\Gamma(2-\alpha)} \sum_{j=0}^{k} \frac{y\left(x_{i}, t_{k+1-j}\right)-y\left(x_{i}, t_{k-j}\right)}{\tau}\left[(j+1)^{1-\alpha}-j^{1-\alpha}\right] \\
& =\frac{u\left(x_{i}\right)}{h^{\beta}} \sum_{j=0}^{i+1} a_{j} y\left(x_{i}-(j-1) h, t_{k+1}\right)-c\left(x_{i}\right) y\left(x_{i}, t_{k+1}\right) \\
& -v\left(x_{i}\right) \frac{y\left(x_{i}, t_{k+1}\right)-y\left(x_{i-1}, t_{k+1}\right)}{h}+b\left(x_{i}, t_{k+1}\right)+O(\tau+h) .
\end{aligned}
$$

Let $u_{i}=u\left(x_{i}\right), v_{i}=v\left(x_{i}\right), c_{i}=c\left(x_{i}\right), c_{i} \cong \tau^{\alpha} \Gamma(2-\alpha) \geq 0$,

$b_{i}^{k}=b\left(x_{i}, t_{k}\right), \quad \sigma_{j}=(j+1)^{1-\alpha}-j^{1-\alpha}, \quad j=0,1,2, \mathrm{~L}, n$;

$r_{i}=$

$\frac{u_{i} \tau^{\alpha}}{h^{\beta}} \Gamma(2-\alpha) \geq 0, p_{i}=\frac{v_{i} \tau^{\alpha}}{h} \Gamma(2-\alpha) \geq 0, g_{1}^{k}=g_{1}\left(t_{k}\right), g_{2}^{k}$ $=g_{2}\left(t_{k}\right), y_{i}^{k}$ represents numerical solution of Eqs. (6)-(8), then we have

$$
\begin{aligned}
& \sum_{j=0}^{k} \sigma_{j}\left(y_{i}^{k+1-j}-y_{i}^{k-j}\right)=-p_{i}\left(y_{i}^{k+1}-y_{i-1}^{k+1}\right)+r_{i} \sum_{j=0}^{i+1} a_{j} y_{i-j+1}^{k+1} \\
& -c_{i} y_{i}^{\otimes 1}+c_{i} b_{i}^{\otimes 1} .
\end{aligned}
$$

Since local truncation error is $O(\tau+h)$, thus the difference scheme is consistent[10]. Eqs. (14) will be repalced by

When $k=0$,

$$
\begin{aligned}
& -r_{i} y_{i+1}^{1}+\left(1+p_{i}-r_{i} a_{1}+c_{i}\right) Q_{i}^{1}-\left(p_{i}+r_{i} a_{2}\right) y_{i-1}^{1}-r_{i} \sum_{j=3}^{i+1} a_{j} y_{i-j+1}^{1} \\
& =y_{i}^{0}+c_{i} b_{i}^{0},
\end{aligned}
$$

when $k>0$,

$$
\begin{aligned}
& -r_{i} y_{i+1}^{k+1}+\left(1+p_{i}-r_{i} a_{1}+c_{i}\right) \mathcal{Y}_{i}^{k+1}-\left(p_{i}+r_{i} a_{2}\right) y_{i-1}^{k+1}-r_{i} \sum_{j=3}^{i+1} a_{j} y_{i-j+1}^{k+1} \\
& =y_{i}^{k}-\sum_{j=0}^{k} \sigma_{j}\left(y_{i}^{k+1-i}-y_{i}^{k-j}\right)=\left(2-2^{1-\alpha}\right) y_{i}^{k}+\sigma_{k} y_{i}^{0}+c b_{i} b^{1}- \\
& \sum_{j=1}^{k-1} y_{i}^{k-j}\left[2(j+1)^{1-\alpha}-(j+2)^{1-\alpha}-j^{1-\alpha}\right] \\
& =d_{1} y_{i}^{k}-\sum_{j=1}^{k-1} y_{i}^{k-j} d_{j+1}+\sigma_{k} y_{i}^{0}+c_{i} b_{i}^{\boldsymbol{O}^{1}} \text {, }
\end{aligned}
$$


where $d_{j}=\sigma_{j-1}-\sigma_{j}, i=1,2, \mathrm{~L}, m-1 ; k=1,2, \mathrm{~L}, n-1$.

\section{Stability AND CONVERGENCE OF The DifFERENCE SCHEME}

Lemma 2.1 For arbitrary real number $a, b, c, d$, we have

$$
-|a|+|b|-|c|-|d| \leq|-a+b-c-d| .
$$

Proof. From $|b|=|-a+b-c-d+a+c+d|$

$$
\leq|-a+b-c-d|+|a|+|c|+|d|,
$$

we obtain $-|a|+|b|-|c|-|d| \leq|-a+b-c-d|$.

Lemma 2.2 (1) $a_{j}>0(j \geq 2)$. (2) For any positive integer $N$, we have $\sum_{j=0}^{N} a_{j}<0$.

Proof. (1) Note that $a_{j}=(-1)^{j}\left(\begin{array}{c}\beta \\ j\end{array}\right)$ and $1<\beta<2$, we know that $a_{j}>0(j \geq 2)$.

(2) Since $(1+x)^{\beta}=\sum_{j=0}^{\infty}\left(\begin{array}{c}\beta \\ j\end{array}\right) x^{j}, x \in[-1,1]$, let $x=-1$, then $\sum_{j=0}^{\infty} a_{j}=0$. From Lemma 2.2(1), we have that

$$
\sum_{j=0}^{N} a_{j}=-\sum_{j=N+1}^{\infty} a_{j}<0
$$

$\operatorname{Lemma2.3}(1) \sum_{j=1}^{n} d_{j}=1-\sigma_{n} ;$ (2) $d_{j}>0, \sigma_{j-1}>\sigma_{j}$.

Proof. (1)From $d_{j}=\sigma_{j-1}-\sigma_{j}, \sigma_{j}=(j+1)^{1-\alpha}-j^{1-\alpha}$, we easily know that $\sum_{j=1}^{n} d_{j}=1-\sigma_{n}$.

(2) Let $h(x)=(x+1)^{1-\alpha}-x^{1-\alpha}(x \geq 1)$, then $h^{\prime}(x)=$ $(1-\alpha)\left[(x+1)^{-\alpha}-x^{-\alpha}\right]<0, \quad$ so $h(x) \quad$ is decreasing function, $d_{j}=\sigma_{j-1}-\sigma_{j}=h(j-1)-h(j)>0$. Therefore, we have that $d_{j}>0, \sigma_{j-1}>\sigma_{j}$.

\section{A. Stability of the difference scheme}

Theorem 2.1 Implicit difference schemes defined by Eqs. (15)-(16) are unconditionally steady for initial value[10].

Proof. Suppose that $y_{i}^{\circ}, y_{i}^{k}$ represent solutions of Eqs. (15)-(16) for initial value $f_{1}(x), f_{2}(x)$ respectively, and $b_{i}^{k}$ is accurate vale, then error $\varepsilon_{i}^{k}=\mathscr{H}_{i}^{\circ}-y_{i}^{k}$ satisfies
When $k=0$,

$$
\begin{aligned}
& -r_{i} \varepsilon_{i+1}^{1}+\left(1+p_{i}-r_{i} a_{1}+c_{i}^{\top}\right) \boldsymbol{\varepsilon}_{i}-\left(p_{i}+r_{i} a_{2}\right) \varepsilon_{i-1}^{1} \\
& -r_{i} \sum_{j=3}^{i+1} a_{j} \varepsilon_{i-j+1}^{1}=\varepsilon_{i}^{0},
\end{aligned}
$$

when $k>0$,

$$
-r_{i} \varepsilon_{i+1}^{k+1}+\left(1+p_{i}-r_{i} a_{1}+c_{i}^{O}\right) \varepsilon_{i}^{k+1}-\left(p_{i}+r_{i} a_{2}\right) \varepsilon_{i-1}^{k+1}
$$

$-r_{i} \sum_{j=3}^{i+1} a_{j} \varepsilon_{i-j+1}^{k+1}=d_{1} \varepsilon_{i}^{k}+\sum_{j=1}^{k-1} d_{j+1} \varepsilon_{i}^{k-j}+\sigma_{k} \varepsilon_{i}^{0}$.

Let $E^{k}=\left(\varepsilon_{1}^{k}, \varepsilon_{2}^{k}, \mathrm{~L}, \varepsilon_{m-1}^{k}\right)^{\prime}$, we prove $\left\|E^{k}\right\|_{\infty} \leq\left\|E^{0}\right\|_{\infty}$ with mathematical induction in the following.

When $k=1$, let $\left|\varepsilon_{l}^{1}\right|=\max _{1 \leq i \leq m-1}\left|\varepsilon_{i}^{1}\right|$. Note that $r_{l}>0$, $p_{l}>0, a_{0}=1, a_{1}=-\beta<0$, we have from Lemma 2.2 that

$$
\begin{aligned}
\left\|E^{1}\right\|_{\infty} & =\left|\varepsilon_{l}^{1}\right| \leq\left|\varepsilon_{l}^{1}\right|+p_{l}\left(\left|\varepsilon_{l}^{1}\right|-\left|\varepsilon_{l-1}^{1}\right|\right)-r_{l} \sum_{j=0}^{l+1} a_{j}\left|\varepsilon_{l}^{1}\right| \\
& \leq-r_{l}\left|\varepsilon_{l+1}^{1}\right|+\left(1+p_{l}-r_{l} a_{1}+c_{i}\right) \ngtr \varepsilon_{l}^{1} \mid \\
& -\left(p_{l}+r_{l} a_{2}\right)\left|\varepsilon_{l-1}^{1}\right|-r_{l} \sum_{j=3}^{l+1} a_{j}\left|\varepsilon_{l-j+1}^{1}\right| .
\end{aligned}
$$

Note that $p_{l}+r_{l} a_{2}>0, r_{l} \sum_{j=3}^{l+1} a_{j}>0,1+p_{l}-r_{l} a_{1}+c_{i}^{\mathscr{O}} \mathbf{9 0}$, and from Lemma 2.1, we further obtain that

$$
\begin{aligned}
& \left|\varepsilon_{l}^{1}\right| \leq \mid-r_{l} \varepsilon_{l+1}^{1}+\left(1+p_{l}-r_{l} a_{1}+c_{i}^{0}\right) \mathcal{Q}_{l}-\left(p_{l}+r_{l} a_{2}\right) \varepsilon_{l-1}^{1} \\
& -r_{l} \sum_{j=3}^{l+1} a_{j} \varepsilon_{l-j+1}^{1}|=| \varepsilon_{l}^{0} \mid \leq\left\|E^{0}\right\|_{\infty} .
\end{aligned}
$$

Thus $\left\|E^{1}\right\|_{\infty}=\varepsilon_{l}^{1} \mid \leq\left\|E^{0}\right\|_{\infty}$.

Assume that we always have $\left\|E^{k}\right\|_{\infty} \leq\left\|E^{0}\right\|_{\infty}$ when $k \leq s$, let $\left|\varepsilon_{l}^{s+1}\right|=\max _{1 \leq i \leq m-1}\left|\varepsilon_{i}^{s+1}\right|$, then when $k=s+1$, we have

$$
\begin{aligned}
\left|\varepsilon_{l}^{s+1}\right| \leq & -r_{l}\left|\varepsilon_{l+1}^{s+1}\right|+\left(1+p_{l}-r_{l} a_{1}+c_{i}\right) \Phi \varepsilon_{l}^{s+1} \mid \\
& -\left(p_{l}+r_{l} a_{2}\right)\left|\varepsilon_{l-1}^{s+1}\right|-r_{l} \sum_{j=3}^{l+1} a_{j}\left|\varepsilon_{l-j+1}^{s+1}\right| \\
& \leq-r_{l} \varepsilon_{l+1}^{s+1}+\left(1+p_{l}-r_{l} a_{1}+c_{i}\right) \mathcal{Q}_{l}^{+1}
\end{aligned}
$$




$$
\begin{aligned}
& -\left(p_{l}+r_{l} a_{2}\right) \varepsilon_{l-1}^{s+1}-r_{l} \sum_{j=3}^{l+1} a_{j} \varepsilon_{l-j+1}^{s+1} \mid \\
= & \left|d_{1} \varepsilon_{l}^{s}+\sum_{j=1}^{s-1} d_{j+1} \varepsilon_{l}^{s-j}+\sigma_{s} \varepsilon_{l}^{0}\right| \\
\leq & d_{1}\left\|E^{s}\right\|_{\infty}+\sum_{j=1}^{s-1} d_{j+1}\left\|E^{s-j}\right\|_{\infty}+\sigma_{s}\left\|E^{0}\right\|_{\infty} \\
\leq & \left(d_{1}+\sum_{j=1}^{s-1} d_{j+1}+\sigma_{s}\right)\left\|E^{0}\right\|_{\infty} \\
= & \left(1-\sigma_{s}+\sigma_{s}\right)\left\|E^{0}\right\|_{\infty}=\left\|E^{0}\right\|_{\infty} .
\end{aligned}
$$

Consequently, the desired result follows.

\section{B. Convergence of the difference scheme}

Suppose that $y\left(x_{i}, t_{k}\right)$ is exact solution of the differential equation at $\left(x_{i}, t_{k}\right)$. Let $e_{i}^{k}=y\left(x_{i}, t_{k}\right)-y_{i}^{k}$, $e^{k}=\left(e_{1}^{k}, e_{2}^{k}, \mathrm{~L}, \quad e_{m-1}^{k}\right)^{\prime}, \quad$ then $\quad y_{i}^{k}=y\left(x_{i}, t_{k}\right)-e_{i}^{k}$, substituting it into Eqs. (15)-(16), and note $e^{0}=0$, we have that

When $k=0$,

$$
-r_{i} e_{i+1}^{1}+\left(1+p_{i}-r_{i} a_{1}+c_{i}^{Y}\right) \mathcal{E}_{i}^{\phi}-\left(p_{i}+r_{i} a_{2}\right) e_{i-1}^{1}
$$

$-r_{i} \sum_{j=3}^{i+1} a_{j} e_{i-j+1}^{1}=R_{i}^{1}$

when $k>0$,

$$
\begin{gathered}
-r_{i} e_{i+1}^{k+1}+\left(1+p_{i}-r_{i} a_{1}+c_{i}\right)\left(\mathcal{E}_{i}^{++1}-\left(p_{i}+r_{i} a_{2}\right) e_{i-1}^{k+1}\right. \\
-r_{i} \sum_{j=3}^{i+1} a_{j} e_{i-j+1}^{k+1}=d_{1} e_{i}^{k}+\sum_{j=1}^{k-1} d_{j+1} e_{i}^{k-j}+R_{i}^{k+1},
\end{gathered}
$$

where $\left|R_{i}^{k}\right| \leq \lambda\left(\tau^{1+\alpha}+\tau^{\alpha} h\right), \lambda$ is a positive constant and it has nothing to do with $h, \tau$.

Theorem 2.2 There is a constant $\lambda>0$ such that

$$
\left\|e^{k}\right\|_{\infty} \leq \sigma_{k-1}^{-1} \lambda\left(\tau^{1+\alpha}+\tau^{\alpha} h\right), k=1,2, \mathrm{~L}, n,
$$

where $\lambda$ has nothing to do with $h, \tau$, and $\left\|e^{k}\right\|_{\infty}=$ $\max _{1 \leq i \leq m-1}\left|e_{i}^{k}\right|$.

Proof. When $k=1$, let $\left|e_{l}^{1}\right|=\max _{1 \leq i \leq m-1}\left|e_{i}^{1}\right|$, then $\left\|e^{1}\right\|_{\infty}=$ $\left|e_{l}^{1}\right|$, we have from Lemma 2.1 that

$$
\left|e_{l}^{1}\right| \leq-r_{l}\left|e_{l+1}^{1}\right|+\left(1+p_{l}-r_{l} a_{1}+c_{i}^{X}\right)\left\lceil e_{l}^{1} \mid\right.
$$

$$
\begin{aligned}
& -\left(p_{l}+r_{l} a_{2}\right)\left|e_{l-1}^{1}\right|-r_{l} \sum_{j=3}^{l+1} a_{j}\left|e_{l-j+1}^{1}\right| \\
& \leq \mid-r_{l} e_{l+1}^{1}+\left(1+p_{l}-r_{l} a_{1}+c_{i}^{\top}\right) \mathcal{E}_{l}^{j}-\left(p_{l}+r_{l} a_{2}\right) e_{l-1}^{1} \\
& -r_{l} \sum_{j=3}^{l+1} a_{j} e_{l-j+1}^{1}|=| R_{l}^{1} \mid \\
& \leq \lambda\left(\tau^{1+\alpha}+\tau^{\alpha} h\right)=\sigma_{0}^{-1} \lambda\left(\tau^{1+\alpha}+\tau^{\alpha} h\right) .
\end{aligned}
$$

Assume that $\left\|e^{s}\right\|_{\infty} \leq \sigma_{s-1}^{-1} \lambda\left(\tau^{1+\alpha}+\tau^{\alpha} h\right)$ when $k \leq s$, let $\left|e_{l}^{s+1}\right|=\max _{1 \leq i \leq m-1}\left|e_{i}^{s+1}\right|$, then when $k=s+1$, we have that

$$
\begin{aligned}
& \left\|e^{s+1}\right\|_{\infty}=\left|e_{l}^{s+1}\right| \\
& \leq d_{1}\left\|e^{s}\right\|_{\infty}+\sum_{j=1}^{s-1} d_{j+1}\left\|e^{s-j}\right\|_{\infty}+\lambda\left(\tau^{1+\alpha}+\tau^{\alpha} h\right) \\
& \leq\left(1+d_{1} \sigma_{s-1}^{-1}+d_{2} \sigma_{s-2}^{-1}+\mathrm{L}+d_{s} \sigma_{0}^{-1}\right) \lambda\left(\tau^{1+\alpha}+\tau^{\alpha} h\right),
\end{aligned}
$$

as in Lemma 2.3, we have found that $\sigma_{j}^{-1} \leq \sigma_{s}^{-1}(j \leq s)$, so we futher obtain

$$
\begin{aligned}
\left\|e^{s+1}\right\|_{\infty} & \leq \sigma_{s}^{-1}\left(\sum_{i=0}^{s-1} d_{i+1}+\sigma_{s}\right) \lambda\left(\tau^{1+\alpha}+\tau^{\alpha} h\right) \\
& =\sigma_{s}^{-1} \lambda\left(\tau^{1+\alpha}+\tau^{\alpha} h\right) .
\end{aligned}
$$

The desired result follows.

$$
\text { Since } \lim _{k \rightarrow \infty} \frac{\sigma_{k}^{-1}}{k^{\alpha}}=\lim _{k \rightarrow \infty} \frac{1}{k^{\alpha} \sigma_{k}}=\lim _{k \rightarrow \infty} \frac{1}{k\left[(1+1 / k)^{1-\alpha}-1\right]}=
$$

$\frac{1}{1-\alpha}$, thus there is a constant $\gamma>0$ such that

$$
\left\|E^{k}\right\|_{\infty} \leq k^{\alpha} \gamma\left(\tau^{1+\alpha}+\tau^{\alpha} h\right)=(k \tau)^{\alpha} \gamma(\tau+h) .
$$

Consequently, we can obtain the following result when $k \tau \leq T$.

Theorem 2.3 Suppose that $y\left(x_{i}, t_{k}\right)$ denotes exact solution at $\left(x_{i}, t_{k}\right), y_{i}^{k}$ is numerical solution of implicit difference scheme, then there exists a constant $\%=T^{\alpha} \gamma>0$ such that

$$
\left|y\left(x_{i}, t_{k}\right)-y_{i}^{k}\right| \leq \mathscr{Y}(\tau+h), 1 \leq i \leq m, 1 \leq k \leq n .
$$

\section{SOURCE TERM INVERSION}

The inverse problem, which is composed of Eqs. (2)-(5), is to solve nonlinear operator equation

$$
A[b(x)]=\psi(x) .
$$


Suppose that $y(b(x) ; x, t)$ denotes solution of Eqs. (2)(4) for $b(x), b_{0}(x)$ denotes a function near $b^{*}(x)$, where $b_{0}(x)=\sum_{i=1}^{n} k_{i}^{0} \psi_{i}(x)=K_{0}^{\mathrm{T}} \Psi(x)$, and $b^{*}(x)$ denotes exact solution of Eqs. (2)-(4), $b(x) \in K, \psi_{1}(x), \psi_{2}(x), \mathrm{L}$, are a group of primary functions on $K$, then a tiny disturbing quantity of $b_{0}(x)$ is

$$
\delta b_{0}(x)=\sum_{i=1}^{n} \delta k_{i}^{0} \psi_{i}(x)=\delta K_{0}^{\mathrm{T}} \Psi(x),
$$

where

$$
\Psi(x)=\left(\psi_{1}(x), \psi_{2}(x), \mathrm{L}, \psi_{n}(x)\right)^{T},
$$

$K^{T}=\left(k_{1}, k_{2}, \mathrm{~L}, k_{n}\right) \in R^{n}$.

Assume that $y\left(b_{1}(x) ; x, t\right)$ denotes the solution of initial boundary value problem for $b_{1}(x)$, where $b_{1}(x)=b_{0}(x)$ $+\delta b_{0}(x)$, using Taylor formula, then we have[6]

$$
\begin{aligned}
& y\left(b_{0}(x)+\delta b_{0}(x) ; x, t\right) \\
& =y\left(b_{0}(x) ; x, t\right)+\nabla_{K_{0}}^{T} y\left(b_{0}(x) ; x, t\right) \cdot \delta K_{0}+o\left(\left\|\delta b_{0}(x)\right\|\right),
\end{aligned}
$$

by using the Tikhonov regularization method, solving $b(x)$ is converted into $\delta K_{0}$, and $\delta K_{0}$ can be determined by local minimum of the following function[9]

$$
\begin{aligned}
& \quad G\left[\delta K_{0}\right]=\left\|y\left(b_{0}(x)+\delta b_{0}(x) ; x, t\right)-y(x, T)\right\|_{L^{2}\left(\partial \Omega^{\prime} \times[0, T]\right)}^{2} \\
& +\alpha S\left[\delta K_{0}\right]=\| y\left(b_{0}(x) ; x, t\right)-y(x, T)+ \\
& \nabla_{K_{0}}^{T} y\left(b_{0}(x) ; x, t\right) \cdot \delta K_{0} \|_{L^{2}\left(\partial \Omega^{\prime} \times[0, T]\right)}^{2}+\alpha S\left[\delta K_{0}\right],
\end{aligned}
$$

where $x \in \partial \Omega^{\prime} \subset \partial \Omega, \alpha$ denotes regularization coefficient, $S\left[\delta K_{0}\right]$ denotes steady functional of $\delta K_{0}$.

Assume that there are discrete points $x_{m}(m=1,2, \mathrm{~L}$, $M)$ on $\partial \Omega^{\prime}, \quad S\left[\delta K_{0}\right]=\delta K_{0}^{T} \delta K_{0}$, then

$$
\begin{aligned}
G\left[\delta K_{0}\right] & =\delta K_{0}^{T} A^{T} A \delta K_{0}+2 \delta K_{0}^{T} A^{T}(P-Q) \\
& +(P-Q)^{T}(P-Q)+\alpha \delta K_{0}^{T} \delta K_{0} .
\end{aligned}
$$

It is easy to prove that solving the local minimum values of Eqs. (19) is equivalent to slove $\left(A^{T} A+\alpha I\right) \delta K_{0}=A^{T}$ $(Q-P)$, so we have

$$
\delta K_{0}=\left(A^{T} A+\alpha I\right)^{-1} A^{T}(Q-P)
$$

where

$$
\begin{gathered}
P=\left[\begin{array}{c}
y\left(b_{0}(x) ; x_{1}, T\right) \\
y\left(b_{0}(x) ; x_{2}, T\right) \\
\mathrm{M} \\
y\left(b_{0}(x) ; x_{M}, T\right)
\end{array}\right], \quad Q=\left[\begin{array}{c}
y_{T}\left(x_{1}\right) \\
y_{T}\left(x_{2}\right) \\
\mathrm{M} \\
y_{T}\left(x_{M}\right)
\end{array}\right], \\
A=\left(a_{i j}\right)_{M \times N}, a_{i j}=\frac{\partial}{\partial k_{j}} y\left(b_{0}(x) ; x_{i}, T\right) .
\end{gathered}
$$

Substituting Eqs. (20) into Eqs. (17), we can obtain $\delta b_{0}(x)$ and a new approximation of the exact solution, $b_{1}(x)=b_{0}(x)+\delta b_{0}(x)$.

Repeating the above, until satisfies the precision requirement.

\section{NUMERICAL EXPERIMENTS}

In order to verify the effectiveness of the algorithm in the source term identification, we do the following numerical experiment[7]. For simplicity, we set part variables as follows

$$
\begin{aligned}
& c(x)=0.05, u(x)=292, v(x)=365, L=4000, T=11, \\
& m=20, n=11, \Delta=[0.01,0.01,0.01] .
\end{aligned}
$$

Where $\Delta$ is the increment of $K$ when computing matrix $A$ from Eqs. (20). Moreover, we always take polynomial function space as primary function space in the following computation, and setting initial boundary condition as follows

$$
\begin{aligned}
& y_{T}(x)=0.057 x+45.6,0 \leq x \leq 4000, \\
& g_{1}(t)=0.724 t^{2}+45.6,0 \leq t \leq 11, \\
& g_{2}(t)=2.2 t^{2}+273.4,0 \leq t \leq 11 .
\end{aligned}
$$

Let $b(x)=1-x$ in the Eqs. (6)-(8), and substituting initial boundary condition, we can obtain $y(X, T)$ by sloving forward problem. And as the additional data, we can do inversion calculation by using the above algorithm. Let initial iteration vector $K_{0}=[1,1,1]$, and iterative termination condition $\delta b(x)<1 e-4$, then we obtain inversion results under different regularization coefficient(see TABLE I), let theta $=1 e-3$, we get inversion results under different initial value(see TABLE II).

TABLE I . THE INVERSION RESULTS UNDER DIFFERENT REGULARIZATION COEFFICIENT

\begin{tabular}{|l|l|l|}
\hline$\theta$ & Iteration times & Results \\
\hline
\end{tabular}




\begin{tabular}{|c|l|lll|}
\hline $1 \mathrm{e}-1$ & 4 & 1.0000 & -1.0000 & 0 \\
$1 \mathrm{e}-2$ & 3 & 1.0000 & -1.0000 & 0 \\
$1 \mathrm{e}-3$ & 3 & 1.0000 & -1.0000 & 0 \\
$1 \mathrm{e}-4$ & 3 & 1.0000 & -1.0000 & 0 \\
\hline
\end{tabular}

TABLE II . THE INVERSION RESULTS UNDER DIFFERENT INITIAL VALUE

\begin{tabular}{|rcl|c|ccl|}
\hline \multicolumn{3}{|c|}{$K_{0}$} & $\begin{array}{c}\text { Iteration } \\
\text { times }\end{array}$ & Results & \\
\hline-100 & -100 & -100 & 4 & 1.0000 & -1.0000 & 0 \\
-10 & -10 & -10 & 3 & 1.0000 & -1.0000 & 0 \\
1 & 1 & 1 & 3 & 1.0000 & -1.0000 & 0 \\
10 & 10 & 10 & 3 & 1.0000 & -1.0000 & 0 \\
100 & 100 & 100 & 4 & 1.0000 & -1.0000 & 0 \\
\hline
\end{tabular}

To better simulate the errors generated by actual data, and verify the effectiveness of the algorithm, we choose the disturbance error $V^{\rho}=V(1+\xi \rho)$, where $\xi \in[-1,1]$, and $\rho>0$ is error level.

According to the above algorithm, we do 8 times numerical experiments, and obtain the inversion results under different error level $\rho$ (see TABLE III). Besides, the comparison of inversion results and exact solution can see Figure 1 when $\rho=0.01$.

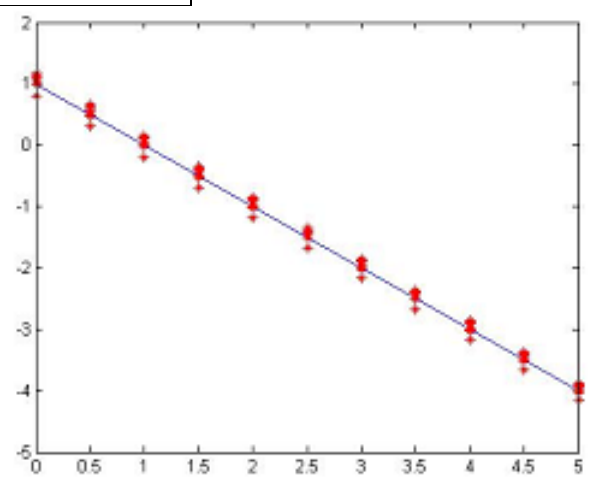

Figure 1. The comparison of inversion results and exact solutions

TABLE III. THE INVERSION RESULTS UNDER DIFFERENT ERROR LEVEL

\begin{tabular}{|c|c|c|c|}
\hline Times & $\rho=0.01$ & $\rho=0.05$ & $\rho=0.1$ \\
\hline 1 & $0.9941-0.9997-0.0000$ & $1.4988-1.0243-0.0000$ & $0.3838-0.9700-0.0000$ \\
2 & $1.1639-1.0080-0.0000$ & $0.3221-0.9670-0.0000$ & $2.3115-1.06390 .0000$ \\
3 & $1.1098-1.00530 .0000$ & $0.8026-0.9903-0.0000$ & $-1.0527-0.9000-0.0000$ \\
4 & $0.9818-0.9991-0.0000$ & $1.9119-1.04440 .0000$ & $-0.5123-0.9264-0.0000$ \\
5 & $0.7984-0.9902-0.0000$ & $1.8730-1.04250 .0000$ & $-0.2448-0.9394-0.0000$ \\
6 & $1.1346-1.00660 .0000$ & $0.8121-0.9909-0.0000$ & $-0.2617-0.9386-0.0000$ \\
7 & $0.9768-0.9989-0.0000$ & $1.8243-1.0401-0.0000$ & $1.4347-1.02120 .0000$ \\
8 & $1.0483-1.00240 .0000$ & $0.0742-0.9549-0.0000$ & $0.0459-0.9535-0.0000$ \\
\hline mean value & $0.9941-1.00130 .0000$ & $1.1399-1.00670 .0000$ & $0.2631-0.9641-0.0000$ \\
\hline
\end{tabular}

Through the above numerical experiment, we find that the inversion results and exact solution are almost the same, and this shows that the above algorithm is feasible and very effective.

\section{REFERENCES}

[1] Andreas Kirsch, An introduction to the mathematical theory of inverse problems, Springer, Karlsruhe, 1996.

[2] Gongsheng Li, Jinqing Liu, et al., A new gradient regularization algorithm for source term inversion in 1D solute transportation with final observations, Appl. Math. Comput, Vol.196,pp. 646-660, 2008.

[3] Yang Zhang, A finite method for fractional partial differential equation,Applied Mathematics and Computation, Vol.215, pp. 524-529, 2009.

[4] Meerschaert M M and Tadjeran C, Finite difference approximations for fractional advection-dispersion flow equations, J. Comput. Appl. Math.,
Vol.172,pp. 65-77, 2004.

[5] Podlubny I, Fractional differential equations, Academic Press, San Diego, 1999 .

[6] Jinqing Liu, Gongsheng Li and $\mathrm{Yu}$ Ma, Gradient—regulation method for determining a pollution source term in groundwater, Journal of Shandong University of Technology(Natural Science Edition), Vol. 21,No.2,pp.17-21,2007.

[7] Gongsheng Li, Yongji Tan and Xiaoqin Wang, Inverse problem method on determining magnitude of groundwater pollution sources, Mathematica Applicata, Vol.18, No.1, pp.92-98, 2005.

[8] Nazheng Sun, Inverse problem in groundwater modeling. Kluwer, Dordrecht, 1994.

[9] Tingyan Xiao, Shengen Yu and Yanfei Wang, Numerial solution of inverse problem, Beijing: Science Press, 2003.

[10] Wensheng Zhang, The finite difference method of partial differential equation in science calculation, Beijing: Higher Education Press, 2006. 